\title{
FORCING INDESTRUCTIBILITY OF SET-THEORETIC AXIOMS
}

\author{
BERNHARD KÖNIG
}

\begin{abstract}
Various theorems for the preservation of set-theoretic axioms under forcing are proved, regarding both forcing axioms and axioms true in the Levy-Collapse. These show in particular that certain applications of forcing axioms require to add generic countable sequences high up in the set-theoretic hierarchy even before collapsing everything down to $\aleph_{1}$. Later we give applications, among them the consistency of MM with $\aleph_{\omega}$ not being Jonsson which answers a question raised during Oberwolfach 2005.
\end{abstract}

\section{INTRODUCTION}

It was a widely held intuition in the early days of proper forcing that a typical application of the Proper Forcing Axiom makes use of a poset of the form $\sigma$-closed $*$ ccc. The usual argument was to collapse the size of all objects to $\aleph_{1}$, then use a ccc-poset to force the desired property to these objects and finally pull everything back into the ground model with the help of the forcing axiom. Later it was realized that forcing axioms have a lot more applications than that. These new developments were heading into two different directions, on the one hand there was the development of semiproper forcing in [15] which lead to the Semiproper Forcing Axiom and later to Martin's Maximum in [5]. On the other hand, even for PFA it was soon realized that there is a large variety of proper forcings that are not of the form $\sigma$-closed $*$ ccc. Interesting examples here are posets that are not $\omega$-proper and it was demonstrated first in [15] and later in [13] that these can be used to good account. The point we are trying to make is slightly different and includes both proper and semiproper forcing notions. We give examples to show that certain applications of forcing axioms can require adding reals or countable sequences even before we collapse the size of the relevant objects to $\aleph_{1}$. The intuition here is that if we collapse without adding countable sequences then our object will have an enumeration

2000 Mathematics Subject Classification. 03E35, 03E50.

Key words and phrases. forcing axioms, transfer principles.

The author acknowledges a grant awarded by the French ministry of research. 
of order-type $\omega_{1}$ whose initial segments are in the ground model. But certain applications exclude such a possibility, most prominently the negation of approachability properties. We just sketched the general direction of this article. Section 2 introduces a wide range of forcing properties that will become important later for the indestructibility theorems. In Section 3 we will give various indestructibility results for forcing axioms, but also indestructibility results for axioms true in the Levy-Collapse of a large cardinal, most notably the axiom of Game Reflection from [7]. Section 4 is a way of applying this technique to Jonsson cardinals and related model-theoretic transfer properties. Among other things, it is shown there that MM is consistent with $\aleph_{\omega}$ not being Jonsson which shoots down a lingering conjecture.

The reader is assumed to have a strong background in set theory. As a general reference we recommend [11] and as a reference regarding proper and semiproper forcing we suggest [2] and [16]. The remaining paragraphs of the introduction will be used to give a short summary of the most important Lemmas and Definitions used in this paper.

1 Definition. If $\Gamma$ is a class of posets then $\mathrm{MA}(\Gamma)$ denotes the statement that whenever $\mathbb{P} \in \Gamma$ and $D_{\xi}\left(\xi<\omega_{1}\right)$ is a collection of dense subsets of $\mathbb{P}$ then there exists a filter $G \subseteq \mathbb{P}$ such that $D_{\xi}$ intersects $G$ for all $\xi<\omega_{1}$. In particular, PFA is MA(proper) and MM is MA(preserving stationary subsets of $\left.\omega_{1}\right)$. The interested reader is referred to [2] and [5] for more history on these forcing axioms.

2 Definition. We would like to remind the reader of the notion of a bounded forcing axiom. Assume that $\lambda$ is a cardinal and $\Gamma$ a class of posets, then the following are equivalent (see [1] and [17]):

(1) For every $\mathbb{P} \in \Gamma$ and a collection $D_{\xi}\left(\xi<\omega_{1}\right)$ of dense subsets of $\mathbb{P}$ of size $\leq \lambda$ there is a filter $G \subseteq \mathbb{P}$ such that $D_{\xi}$ intersects $G$ for all $\xi<\omega_{1}$.

(2) For every $A \subseteq \lambda$ and every $\Sigma_{1}$-formula $\varphi(x)$, if there is some $\mathbb{P} \in \Gamma$ such that $\Vdash_{\mathbb{P}} \varphi(A)$ then there are stationarily many $M \prec \mathrm{H}_{\lambda^{+}}$of size $\aleph_{1}$ containing $A$ such that $\mathrm{H}_{\lambda^{+}} \models \varphi\left(\pi_{M}(A)\right)$, where $\pi_{M}$ is the transitive collapse of $M$.

So let us denote the equivalent statements (1) and (2) by $\operatorname{MA}(\Gamma, \lambda)$ and for simplicity we write $\operatorname{PFA}(\lambda)$ for $\operatorname{MA}($ proper, $\lambda)$ and $\operatorname{MM}(\lambda)$ for MA(preserving stationary subsets of $\left.\omega_{1}, \lambda\right)$. The axioms $\operatorname{PFA}\left(\omega_{1}\right)$ and $\operatorname{MM}\left(\omega_{1}\right)$ are often denoted by BPFA and BMM respectively. If $\Gamma$ is any class of posets, we also write $\operatorname{PFA}(\Gamma)$ for $\mathrm{MA}$ (proper and in $\Gamma$ ) and similarly with $\mathrm{MM}(\Gamma)$.

3 Definition. The approachability property for $\kappa\left(\mathrm{AP}_{\kappa}\right)$ is the statement that there is a sequence $\left(C_{\alpha}: \alpha<\kappa^{+}\right)$such that for any $\alpha<\kappa^{+}$: 
(a) $C_{\alpha} \subseteq \kappa^{+}$, otp $C_{\alpha} \leq \kappa$,

and there is a club $C \subseteq \lim \left(\kappa^{+}\right)$such that for every $\gamma \in C$ :

(b) $C_{\gamma} \subseteq \gamma$ is club,

(c) the initial segments of $C_{\gamma}$ are enumerated before $\gamma$, i.e. $\forall \alpha<\gamma \exists \beta<\gamma C_{\gamma} \cap \alpha=C_{\beta}$.

A straightforward argument shows that $\mathrm{AP}_{\kappa}$ follows from either $\square_{\kappa}$ or from the cardinal arithmetic $\kappa^{<\kappa}=\kappa$.

We use an abbreviation in the context of elementary embeddings: $j: M \longrightarrow N$ means that $j$ is a non-trivial elementary embedding from $M$ into $N$ such that $M$ and $N$ are transitive. The critical point of such an embedding, i.e. the first ordinal moved by $j$, is denoted by $\operatorname{cp}(j)$. We write $j x$ for $j(x)$ in a context where too many parentheses might be confusing. Let us remind ourselves of the well-known extension Lemma for elementary embeddings first noticed by Silver:

4 Lemma (Extension Lemma). Let $j: M \longrightarrow N$ and assume that $G \subseteq \mathbb{P}$ is generic over $M$ and $K \subseteq j(\mathbb{P})$ generic over $N$. If $j^{\prime \prime} G \subseteq K$ then there is a unique extension $j^{*}: M[G] \longrightarrow N[K]$ of $j$ such that $j^{*}(G)=K$.

Proof. For each $\mathbb{P}$-name $\dot{\tau}$ simply let $j^{*}(\dot{\tau}[G])=j(\dot{\tau})[K]$.

5 Definition. Recall that the model-theoretic transfer property

$$
\left(\lambda_{1}, \lambda_{0}\right) \rightarrow\left(\kappa_{1}, \kappa_{0}\right)
$$

means that every structure $\left(\lambda_{1}, \lambda_{0}, f_{i}\right)_{i<\omega}$ has an elementary substructure of the form $\left(A_{1}, A_{0}, f_{i}\right)_{i<\omega}$, where $\left|A_{1}\right|=\kappa_{1}$ and $\left|A_{2}\right|=\kappa_{0}$. The relation $\left(\omega_{2}, \omega_{1}\right) \rightarrow\left(\omega_{1}, \omega\right)$ is usually called Chang's conjecture. A cardinal $\mu$ is called $\kappa$-Rowbottom if for all $\lambda<\mu$ we have $(\mu, \lambda) \rightarrow(\mu,<\kappa)$. A cardinal $\mu$ is called Jonsson if every algebra of size $\mu$ has a proper subalgebra of size $\mu$.

We also need the following well-known Lemmas:

6 Lemma. Let $\lambda$ be regular and assume that $\mathcal{P}$ is a $\sigma$-closed poset of size $\lambda^{\aleph_{0}}$ that collapses $\lambda^{\aleph_{0}}$ to $\aleph_{1}$. Then $\mathcal{P}$ is forcing-isomorphic to $\operatorname{Col}\left(\aleph_{1}, \lambda\right)$.

7 Lemma. Assume that $\mathcal{P}$ is strategically $\sigma$-closed and $\lambda \geq|\mathcal{P}|$. Then $\mathcal{P}$ completely embeds into $\operatorname{Col}\left(\aleph_{1}, \lambda\right)$.

Proof. Clearly, $\mathcal{P}$ completely embeds into $\mathcal{P} \times \operatorname{Col}\left(\aleph_{1}, \lambda\right)$. Results in [4] imply that $\mathcal{P} \times \operatorname{Col}\left(\aleph_{1}, \lambda\right)$ is $\sigma$-closed. Finally, Lemma 6 concludes that $\mathcal{P} \times \operatorname{Col}\left(\aleph_{1}, \lambda\right) \cong \operatorname{Col}\left(\aleph_{1}, \lambda\right)$ and we are done. 


\section{2. $\omega_{2}$-CLOSURE PROPERTIES}

In this section we introduce five different properties of forcings which all entail that no new $\omega_{1}$-sequences be added. We give a small overview before defining them one by one, the following list is increasing in logical strength:

(1) $\omega_{2}$-distributive

(2) weakly $\left(\omega_{1}+1\right)$-game-closed

(3) strongly $\left(\omega_{1}+1\right)$-game-closed

(4) $\omega_{2}$-closed

(5) $\omega_{2}$-directed-closed

So let us start with distributivity. A poset $\mathbb{P}$ is called $\kappa$-distributive if the intersection of less than $\kappa$ many dense open subsets is again dense open. Note that this is equivalent to saying that $\mathbb{P}$ adds no new sequences of length less than $\kappa$. It will become clear later why we are mostly interested in the case $\kappa=\omega_{2}$. We have the following proposition:

8 Lemma. Let $\lambda \geq \aleph_{1}$. The bounded forcing axiom $\operatorname{MM}(\lambda)$ is preserved by $\lambda^{+}$-distributive forcings. Moreover, $\operatorname{PFA}(\lambda)$ is preserved by proper $\lambda^{+}$-distributive forcings.

Proof. This follows simply from the fact that $\lambda^{+}$-distributive forcings add no new elements to $\mathrm{H}_{\lambda^{+}}$, so check that (2) of Definition 2 holds in any (proper) $\lambda^{+}$-distributive extension. For the $\operatorname{MM}(\lambda)$-argument, note that $\lambda^{+}$-distributive forcings preserve stationary subsets of $\omega_{1}$.

Now recall longer versions of the Banach-Mazur game on a partial ordering $\mathbb{P}$ :

\begin{tabular}{c|ccccc} 
Empty & $p_{0}$ & $p_{2}$ & $\ldots$ & $p_{\xi}$ & $\ldots$ \\
\hline Nonempty & $p_{1}$ & $p_{3}$ & $\ldots$ & $p_{\xi+1}$ & $\ldots$
\end{tabular}

where $p_{\xi}(\xi<\alpha)$ is descending in $\mathbb{P}$ and Nonempty wins the game of length $\alpha$ if he can play $\alpha$ times.

9 Definition. A poset $\mathbb{P}$ is called weakly $\alpha$-game-closed if Player Nonempty has a winning strategy in the Banach-Mazur game of length $\alpha$, where Nonempty is allowed to play at limit stages. $\mathbb{P}$ is called strongly $\alpha$-game-closed if Player Nonempty has a winning strategy in the same game except where Empty is allowed to play at limit stages.

It is clear that strongly $(\kappa+1)$-game-closed posets are also weakly $(\kappa+1)$-game-closed. Remember that the standard forcing to add a $\square_{\kappa}$-sequence with initial segments is weakly $(\kappa+1)$-game-closed. An $\mathrm{AP}_{\kappa}$-sequence can be added with a considerably milder forcing. The following crucial fact is originally from [20]: 
10 Lemma. For all cardinals $\kappa$ there is a strongly $(\kappa+1)$-game-closed forcing $\mathbb{A}_{\kappa}$ that adds an $\mathrm{AP}_{\kappa}$-sequence.

11 Definition. Assume for the following that $\mathbb{P}$ is strongly $\left(\omega_{1}+1\right)$ game-closed. Let us fix a winning strategy $\sigma$ for Nonempty in the Banach-Mazur game on $\mathbb{P}$. Instead of forcing with $\mathbb{P}$, we could also add a play of the game generically. Then this play induces a generic filter for $\mathbb{P}$. Define

$$
\mathbb{R}=\left\{\left\langle p_{\xi}: \xi \leq \gamma\right\rangle: \gamma<\omega_{1} \text { and } p_{\xi}(\xi \leq \gamma) \text { is a partial } \sigma \text {-play }\right\} .
$$

If $s=\left\langle p_{\xi}: \xi \leq \gamma\right\rangle \in \mathbb{R}$ is such a partial play, we also denote the maximal condition $p_{\gamma}$ by $p_{s}$. The ordering on $\mathbb{R}$ is usual extension. Note that $\mathbb{R}$ is $\sigma$-closed and contains $\mathbb{P}$ as a complete subalgebra by the projection mapping $i(s)=p_{s}$. Yet, it is a much stronger forcing: $\mathbb{R}$ will typically collapse the cardinality of $\mathbb{P}$ to $\aleph_{1}$.

12 Lemma. Using the notation of Definition 11, if $G \subseteq \mathbb{P}$ is generic then $\mathbb{R} / \mathbb{P}=\left\{s \in \mathbb{R}: p_{s} \in G\right\}$ is $\sigma$-closed.

Proof. Suppose $s_{n}(n<\omega)$ is a descending sequence in $\mathbb{R} / \mathbb{P}$ and $\gamma$ the length of the union $\bigcup_{n<\omega} s_{n}$. Then $q=\inf _{n<\omega} p_{s_{n}}$ is in $G$ and

$$
s=\bigcup_{n<\omega} s_{n} \cup\{(\gamma, q)\}
$$

is still a partial play according to $\sigma$.

13 Lemma. Assume $\mathbb{P}$ is strongly $\left(\omega_{1}+1\right)$-game-closed and $\lambda \geq|\mathbb{P}|^{\aleph_{0}}$. Then $\operatorname{Col}\left(\omega_{1}, \lambda\right) / \mathbb{P}$ is $\sigma$-closed.

Proof. Let $\mathbb{R}$ be as before in Definition 11. The Lemma follows from the following calculation:

$$
\begin{aligned}
\operatorname{Col}\left(\aleph_{1}, \lambda\right) / \mathbb{P} & \cong\left(\mathbb{R} \times \operatorname{Col}\left(\aleph_{1}, \lambda\right)\right) / \mathbb{P} \quad(\text { by Lemma } 6) \\
& \cong(\mathbb{R} / \mathbb{P}) \times \operatorname{Col}\left(\aleph_{1}, \lambda\right)
\end{aligned}
$$

and this last product is $\sigma$-closed by Lemma 12 .

Lemma 13 points out the crucial difference between strongly and weakly game-closed forcings: the quotient $\operatorname{Col}\left(\aleph_{1}, \lambda\right) / \mathbb{P}$ will generally not be $\sigma$-closed if $\mathbb{P}$ is only weakly $\left(\omega_{1}+1\right)$-game-closed.

Finally, we introduce the two remaining notions listed at the beginning of the section. For an infinite cardinal $\kappa, \mathbb{P}$ is called $\kappa$-closed if any $\mathbb{P}$-descending chain of length less than $\kappa$ has a lower bound in $\mathbb{P}$. The poset $\mathbb{P}$ is called $\kappa$-directed-closed if it is closed under directed subsets of size less than $\kappa$. [8] proves that PFA is preserved by $\omega_{2}$-closed forcings and [12] that MM is preserved by $\omega_{2}$-directed-closed forcings. 


\section{INDESTRUCTIBILITY OF SET-THEORETIC AXIOMS}

a. Forcing axioms. This section should be seen as a continuation of work started in [8]. There it was shown that PFA implies failure of the approachability property at $\aleph_{1}$. While we do not repeat the full proof here, it is interesting to mention that PFA is applied to a poset $\mathbb{Q}_{0} * \mathbb{Q}_{1} * \mathbb{Q}_{2}$ in this argument, where $\mathbb{Q}_{0}$ adds a Cohen real, $\mathbb{Q}_{1}$ is a collapse with countable conditions, and $\mathbb{Q}_{2}$ is specializing a tree of size $\aleph_{1}$. The curious fact about the proof is that it seems necessary for technical reasons to add the Cohen real right at the start. In the following, we want to argue that the Cohen real is absolutely necessary. We introduce the notion of a $\Sigma$-collapsing poset $\mathbb{Q}$ which means that $\mathbb{Q}$ can be split up into two parts, where the first collapses everything in sight without adding countable sequences, while the second is arbitrary. Almost all known applications of PFA or MM are using $\Sigma$-collapsing posets. This section wants to point out the few arguments where the forcing axiom for $\Sigma$-collapsing posets is not enough even though the full forcing axiom suffices. In other words, in the presented examples it is absolutely necessary to add countable sequences before collapsing everything to $\aleph_{1}$.

14 Definition. A poset $\mathbb{Q}$ is called $\Sigma$-collapsing if it is of the form $\mathbb{Q}=\mathbb{Q}_{0} * \mathbb{Q}_{1}$, where

(1) $\mathbb{Q}_{0}$ is $\aleph_{1}$-distributive and

(2) $\vdash_{\mathbb{Q}_{0}}\left|\mathbb{Q}_{1}\right| \leq \aleph_{1}$

The class of $\Sigma$-collapsing posets that preserve stationary subsets of $\omega_{1}$ is denoted by $\Gamma_{\Sigma}$.

The point of this definition is that a $\Sigma$-collapsing poset will typically collapse its own cardinality to $\aleph_{1}$ without adding countable sequences. The final segment $\mathbb{Q}_{1}$ is allowed to be anything of size at most $\aleph_{1}$ though. Notice that, in the context of forcing axioms, the class $\Gamma_{\Sigma}$ in particular contains all posets that are

- $\aleph_{1}$-distributive (take $\mathbb{Q}_{1}$ to be trivial).

- proper not adding reals (this implies $\aleph_{1}$-distributivity).

- $\sigma$-closed * ccc (since we can assume the ccc-poset to be of size at most $\aleph_{1}[11$, p.62]).

For example, $\operatorname{PFA}\left(\Gamma_{\Sigma}\right)$ implies the axiom MRP from [13] and $\operatorname{MM}\left(\Gamma_{\Sigma}\right)$ implies SRP (see e.g. [19]). As mentioned in the introduction, many classical applications of PFA are actually consequences of the forcing axiom for posets of the form $\sigma$-closed $*$ ccc and all these are also included in $\operatorname{PFA}\left(\Gamma_{\Sigma}\right)$. Before we prove the main theorem of this section, 
we reproduce the following Lemma. The proof is actually a nice exercise but can also be looked up in [10].

15 Lemma. Let $\lambda$ be regular uncountable. Assume that $\mathbb{R}$ is $\lambda$-closed, $A$ a relation on $\lambda$ and $\varphi$ a $\Sigma_{1}^{1}$-sentence. If there is a condition $r \in \mathbb{R}$ such that $r \Vdash_{\mathbb{R}} "(\lambda, A) \models \varphi$ " then $(\lambda, A) \models \varphi$.

Let us prove the main preservation result. We show that strongly $\left(\omega_{1}+1\right)$-game-closed forcings preserve the fragment of MM that contains all the $\Sigma$-collapsing posets. This will later be used for interesting new independence results.

16 Theorem. Assume that $V \models \operatorname{MM}\left(\Gamma_{\Sigma}\right)$ and $\mathbb{P}$ is strongly $\left(\omega_{1}+1\right)$ game-closed. Then $V^{\mathbb{P}} \models \operatorname{MM}\left(\Gamma_{\Sigma}\right)$.

Proof. Assume that

(3.1) $\vdash_{\mathbb{P}} " \mathbb{Q}$ is $\Sigma$-collapsing and preserves stationary subsets of $\omega_{1}$,"

where $\mathbb{Q}_{0} * \mathbb{Q}_{1}$ witnesses that $\mathbb{Q}$ is $\Sigma$-collapsing and $\mathbb{Q}$ is a $\mathbb{P}$-name for a partial ordering. Let $\dot{\tau}_{\xi}\left(\xi<\omega_{1}\right)$ be a sequence of $\mathbb{P}$-names for dense subsets of $\mathbb{Q}$ and define the dense subsets of $\mathbb{P} * \mathbb{Q}$ :

$$
D_{\xi}=\left\{(p, q): p \Vdash_{\mathbb{P}} q \in \dot{\tau}_{\xi}\right\} .
$$

Now remember from the definitions that

$$
\vdash_{\mathbb{P} * \mathbb{Q}_{0}}\left|\mathbb{Q}_{1}\right| \leq \aleph_{1}
$$

and recall the poset $\mathbb{R}$ from Definition 11 which is induced by $\mathbb{P}$. It was shown in Lemma 12 that $\mathbb{R} / \mathbb{P}$ is $\sigma$-closed.

16.1 Claim. $\mathbb{Q}_{1}$, as a forcing notion in $V^{\mathbb{P} * \mathbb{Q}_{0}}$, preserves all stationary subsets of $\omega_{1}$ in $V^{\mathbb{P}}$.

Proof. This simply follows from the fact that $\mathbb{Q}=\mathbb{Q}_{0} * \mathbb{Q}_{1}$ preserves all stationary subsets of $\omega_{1}$ in $V^{\mathbb{P}}$ by $(3.1)$.

16.2 Claim. The iteration $\mathbb{P} * \mathbb{Q}_{0} * \mathbb{R} / \mathbb{P} * \mathbb{Q}_{1}$ preserves stationary subsets of $\omega_{1}$.

Proof. Let $E \subseteq \omega_{1}$ be stationary. Clearly, the stationarity of $E$ is preserved in the three-step iteration $\mathbb{P} * \mathbb{Q}_{0} * \mathbb{P} / \mathbb{R}$. Now assume towards a contradiction that $\mathbb{Q}_{1}$, as a forcing notion in $V^{\mathbb{P} * \mathbb{Q}_{0} * \mathbb{R} / \mathbb{P}}$, would destroy the stationarity of $E$. Then in $V^{\mathbb{P}^{*} \mathbb{Q}_{0}}$

$$
\Vdash_{\mathbb{R} / \mathbb{P}} \text { "there is a } \mathbb{Q}_{1} \text {-name } \dot{C} \text { for a club disjoint from } E \text {." }
$$

Using density arguments, it is straightforward to check that the quoted statement in (3.4) is $\Sigma_{1}^{1}$ over the structure $\left(\omega_{1} \cup \mathbb{Q}_{1}, \in, E, \leq_{\mathbb{Q}_{1}}\right)$. So we can apply Lemma 15 and conclude that in $V^{\mathbb{P} * \mathbb{Q}_{0}}$

$$
\text { there is a } \mathbb{Q}_{1} \text {-name } \dot{C} \text { for a club disjoint from } E \text {. }
$$


But (3.5) says that $\mathbb{Q}_{1}$, as a forcing notion in $V^{\mathbb{P} * \mathbb{Q}_{0}}$, destroys the stationarity of $E$. This contradicts Claim 16.1 so we finished the proof of Claim 16.2.

Now use the forcing axiom in the ground model to get a filter

$$
G * H_{0} * K * H_{1} \subseteq \mathbb{P} * \mathbb{Q}_{0} * \mathbb{R} / \mathbb{P} * \mathbb{Q}_{1}
$$

that is sufficiently generic, in particular for all dense sets $D_{\xi}\left(\xi<\omega_{1}\right)$.

16.3 Claim. The filter $G$ extends to a condition $q$ in $\mathbb{P}$.

Proof. This is using the fact that $K$ yields a play of the Banach-Mazur game of length $\omega_{1}+1$ that exhausts $G$ in the sense that all elements of $G$ are refined during that play. But this play, given by $K$, follows Nonempty's winning strategy so there is a condition $q$ stronger than every condition in $G$.

Claim 16.3 finishes the proof since

$$
q \Vdash_{\mathbb{P}} " H_{0} * H_{1} \subseteq \mathbb{Q}_{0} * \mathbb{Q}_{1} \text { is generic for } \dot{\tau}_{\xi}\left(\xi<\omega_{1}\right) . "
$$

To illustrate the significance of Theorem 16, we turn to the following theorem of Magidor (see [3]).

17 Theorem. MM implies the failure of $\mathrm{AP}_{\aleph_{\omega}}$.

In his proof, Magidor applies a forcing that shoots a new $\omega$-sequence through $\aleph_{\omega}$. A Corollary of Theorem 16 explains why it is necessary in his argument to add a new countable sequence high up in the settheoretic hierarchy:

18 Corollary. The following theory is consistent:

$$
\operatorname{MM}\left(\aleph_{\omega}\right)+\operatorname{MM}\left(\Gamma_{\Sigma}\right)+\operatorname{AP}_{\aleph_{\omega}} .
$$

Proof. We add an $\mathrm{AP}_{\aleph_{\omega}}$-sequence to a model of MM. The corollary now follows from Lemmas 8,10 and Theorem 16.

It has already been proved that PFA $+\mathrm{AP}_{\aleph_{\omega}}$ is consistent. See [3] for more history on that. Next we investigate the $\Sigma$-collapsing fragment of PFA.

19 Corollary. Con $\left(\mathrm{BPFA}+\operatorname{PFA}\left(\Gamma_{\Sigma}\right)+\mathrm{AP}_{\aleph_{1}}\right)$.

Proof. By Lemmas 8,10 and Theorem 16 since $\mathbb{A}_{\aleph_{1}}$ is strategically $\sigma$ closed and therefore proper.

But PFA implies the failure of $\mathrm{AP}_{\aleph_{1}}$ [8], so we get: 
20 Corollary. BPFA and $\mathrm{PFA}\left(\Gamma_{\Sigma}\right)$ together do not imply full PFA.

For transparency, we include a small chart that sums up the results in this area. The class $\Gamma_{\text {cov }}$ is the collection of all posets that preserve stationary subsets of $\omega_{1}$ and have the covering property, i.e. every countable set of ordinals in the extension can be covered by a countable set in the ground model. Note that $\Gamma_{\Sigma}$ is a proper subset of $\Gamma_{\text {cov }}$.

\begin{tabular}{l|l} 
If $V \models \mathrm{MM}$ and $\mathbb{P}$ is $\ldots$ & then $\ldots$ is true in $V^{\mathbb{P}}$. \\
\hline & $\mathrm{BMM}$ \\
(1) $\omega_{2}$-distributive & $\mathrm{BMM}+$ saturation of $\mathrm{NS}_{\omega_{1}}$ \\
(2) weakly $\left(\omega_{1}+1\right)$-game-closed & $\mathrm{BMM}+\mathrm{MM}\left(\Gamma_{\Sigma}\right)$ \\
(4) $\omega_{2}$-closed & $\mathrm{BMM}+\mathrm{MM}\left(\Gamma_{\text {cov }}\right)$ \\
(5) $\omega_{2}$-directed-closed & $\mathrm{MM}$
\end{tabular}

In the table above, (1) is Lemma $8,(2)$ is in [18], (3) is Theorem $16,(4)$ is in [8], and (5) is folklore but can be looked up in [12]. The papers [8] and [9] give counterexamples which show that the results in this chart are basically optimal. For example, it is shown in [8] that adding an $\mathrm{AP}_{\aleph_{1}}$-sequence with a strongly $\left(\omega_{1}+1\right)$-game-closed forcing makes PFA (and therefore $\operatorname{MM}\left(\Gamma_{\text {cov }}\right)$ ) fail in the extension. In [9], an $\omega_{2}$-closed forcing is constructed which makes MM fail in the extension. More details on this last fact can actually be found in Section 4b of this paper.

b. Levy-Collapse. There are similar preservation results for statements true in the Levy-Collapse of a large cardinal. In [7], an attempt was made to axiomatize the theory of the Levy-Collapse with the help of a reflection principle that is in the style of the well-investigated reflection principles for stationary sets and is actually a strengthening of these. This axiom, the Game Reflection Principle or GRP for short, proves all the typical statements known to hold in the Levy-Collapse and is formulated in terms of games. We need the following notions from [7]:

21 Definition. Let $\theta$ be a regular cardinal.

(1) (The Games) If $\mathcal{A} \subseteq{ }^{<\omega_{1}} \theta$ then the game $\mathbb{G}(\mathcal{A})$ has length $\omega_{1}$ and is played as follows:

\begin{tabular}{c|cccccc} 
I & $\alpha_{0}$ & $\alpha_{1}$ & $\ldots$ & $\alpha_{\xi}$ & $\alpha_{\xi+1}$ & $\ldots$ \\
\hline II & $\beta_{0}$ & $\beta_{1}$ & $\ldots$ & $\beta_{\xi}$ & $\beta_{\xi+1}$ & $\ldots$
\end{tabular}

both players I and II play ordinals below $\theta$ and

II wins iff $\left\langle\alpha_{\xi}, \beta_{\xi}: \xi<\omega_{1}\right\rangle \in[\mathcal{A}]$ 
where $[\mathcal{A}]=\left\{f \in{ }^{\omega_{1}} \theta: f\left\lceil\xi \in \mathcal{A}\right.\right.$ for all $\left.\xi<\omega_{1}\right\}$.

(2) (Restricted Games) For $B \subseteq \mathrm{H}_{\lambda}$, define the game $\mathbb{G}^{B}(\mathcal{A})$ by letting the winning conditions be the same as in $\mathbb{G}(\mathcal{A})$ but imposing the restriction on both players to play ordinals in $B \cap \theta$.

(3) ( $\epsilon$-Approachability) A substructure $M \prec \mathrm{H}_{\lambda}$ of size $\aleph_{1}$ is called $\epsilon$-approachable if it is the limit of an $\epsilon$-chain of countable elementary substructures, i.e. there is an $\epsilon$-chain $\left\langle M_{\xi}: \xi<\omega_{1}\right\rangle$ with $M=\bigcup_{\xi<\omega_{1}} M_{\xi}$. We denote the set of all $\epsilon$-approachable substructures of $\mathrm{H}_{\lambda}$ of size $\aleph_{1}$ by $\mathrm{EA}_{\lambda}$ and we drop the subscript if it is clear from the context.

22 Definition. The Game Reflection Principle or GRP is the following statement:

Let $\theta$ be regular, $\mathcal{A} \subseteq{ }^{<\omega_{1}} \theta$ and $\lambda$ much larger than $\theta$. If II has a winning strategy in the game $\mathbb{G}^{M}(\mathcal{A})$ for every $M \in \mathrm{EA}_{\lambda}$, then II has a winning strategy in $\mathbb{G}(\mathcal{A})$.

We have the following two theorems from [7]:

23 Theorem. GRP is equivalent to saying that $\omega_{2}$ is generically supercompact by $\sigma$-closed forcing, i.e. for every regular $\lambda$ there is $\mathbb{P} \in \Gamma$ such that $V^{\mathbb{P}}$ supports $j: V \longrightarrow M$ with $\operatorname{cp}(j)=\omega_{2}, j\left(\omega_{2}\right)>\lambda$, and $j^{\prime \prime} \lambda \in M$.

24 Theorem. Assume that $\kappa$ is supercompact. Then

$$
V^{\mathrm{Coll}\left(\omega_{1},<\kappa\right)} \models \mathrm{GRP} .
$$

Theorem 24 is really contained in the stronger Theorem 25, so we postpone the proof. The following is the preservation argument:

25 Theorem. Assume that $\kappa$ is supercompact and $\dot{\mathbb{P}}$ is a $\operatorname{Coll}\left(\omega_{1},<\kappa\right)$ name for a strongly $\left(\omega_{1}+1\right)$-game-closed partial ordering. Then

$$
V^{\mathrm{Coll}\left(\omega_{1},<\kappa\right) * \dot{\mathbb{P}}} \models \mathrm{GRP} .
$$

Proof. By Theorem 23 it suffices to show that $\omega_{2}$ is generically supercompact by $\sigma$-closed forcing. For any regular $\lambda$ fix $j: V \longrightarrow M$ such that $\operatorname{cp}(j)=\kappa, j(\kappa)>\lambda$, and $j^{\prime \prime} \lambda \in M$. Without restriction, $\lambda>|\dot{\mathbb{P}}|$.

25.1 Claim. $\Vdash_{\operatorname{Coll}\left(\omega_{1},<\kappa\right)} \operatorname{Coll}\left(\omega_{1},[\kappa, j \kappa)\right) / \dot{\mathbb{P}}$ is $\sigma$-closed.

Proof. Note first that this makes sense because $j \kappa>\lambda>|\dot{\mathbb{P}}|$ and therefore it is forced by $\operatorname{Coll}\left(\omega_{1},<\kappa\right)$ that $\dot{\mathbb{P}}$ is a complete subalgebra of $\operatorname{Coll}\left(\omega_{1},[\kappa, j \kappa)\right)$. This last statement is basically Lemma 7 . The claim now follows from Lemma 13. 
Now set $\mathcal{Q}=\operatorname{Coll}\left(\omega_{1},<\kappa\right) * \dot{\mathbb{P}}$. Standard arguments yield

$$
\begin{aligned}
j \mathcal{Q} & =j\left(\operatorname{Coll}\left(\omega_{1},<\kappa\right) * \dot{\mathbb{P}}\right) \\
& =\operatorname{Coll}\left(\omega_{1},<j \kappa\right) * j \dot{\mathbb{P}} \\
& =\operatorname{Coll}\left(\omega_{1},<\kappa\right) * \operatorname{Coll}\left(\omega_{1},[\kappa, j \kappa)\right) * j \dot{\mathbb{P}} \\
& =\operatorname{Coll}\left(\omega_{1},<\kappa\right) * \dot{\mathbb{P}} * \operatorname{Coll}\left(\omega_{1},[\kappa, j \kappa)\right) / \dot{\mathbb{P}} * j \dot{\mathbb{P}} \\
& =\mathcal{Q} * \operatorname{Coll}\left(\omega_{1},[\kappa, j \kappa)\right) / \dot{\mathbb{P}} * j \dot{\mathbb{P}} \\
& =\mathcal{Q} * j \mathcal{Q} / \mathcal{Q} .
\end{aligned}
$$

By the Extension Lemma 4 we can, in $V^{j \mathcal{Q}}$, extend $j$ to

$$
j^{*}: V^{\mathcal{Q}} \longrightarrow M^{j \mathcal{Q}} \text {. }
$$

Notice first that $j^{\prime \prime} \lambda \in M^{j \mathcal{Q}}$. Finally, it holds in the model $V^{\mathcal{Q}}$ that $\omega_{2}$ is generically $\lambda$-supercompact by the forcing $j \mathcal{Q} / \mathcal{Q}$. But remember that

$$
j \mathcal{Q} / \mathcal{Q}=\operatorname{Coll}\left(\omega_{1},[\kappa, j \kappa)\right) / \dot{\mathbb{P}} * j \dot{\mathbb{P}}
$$

is $\sigma$-closed by Claim 25.1.

Note that GRP implies $\mathrm{CH}$ [7] and therefore $\mathrm{AP}_{\aleph_{1}}$. So Theorem 25 tells us nothing if we take $\dot{\mathbb{P}}$ to be the usual forcing that adds an $\mathrm{AP}_{\aleph_{1}}$-sequence. But we can get the following interesting corollary:

26 Corollary. Con $\left(\mathrm{GRP}+\mathrm{AP}_{\aleph_{\omega}}\right)$.

Proof. By Lemma 10 and Theorem 25.

Corollary 26 is interesting because Shelah [14] has shown that if $\kappa$ is supercompact then $\mathrm{AP}_{\kappa^{+\omega}}$ fails. This contrasts the above result in the sense that generic supercompactness of $\omega_{2}$ by $\sigma$-closed forcing does not imply that $\mathrm{AP}_{\aleph_{\omega}}$ fails. To end the section, let us remark that weakly $\left(\omega_{1}+1\right)$-game-closed forcings can introduce square-sequences and this would violate GRP in a very strong fashion [7]. So we cannot hope to have a preservation theorem for weakly $\left(\omega_{1}+1\right)$-game-closed forcings.

\section{Kurepa-TrEes}

a. Jonsson cardinals. Let us go back to Theorem 17 for a while. Magidor's argument that MM implies failure of the approachability property at $\aleph_{\omega}$ has raised hopes that MM can provide a good picture of the combinatorics of the cardinal $\aleph_{\omega}$. Even earlier [5], it was shown that MM implies SCH which puts severe restrictions on the cardinal arithmetic at $\aleph_{\omega}$. In the light of all this, it seemed possible that MM implies $\aleph_{\omega}$ is Jonsson which would solve an old question. We give an argument to refute this last implication, i.e. we show that MM is 
consistent with $\aleph_{\omega}$ not being Jonsson. This answers a question raised during the Oberwolfach set theory meeting in 2005. The following theorem is well-known, see for example [6].

27 Theorem. Assume that $\mu$ is the least Jonsson cardinal. Then $\mu$ is $\lambda$-Rowbottom for some $\lambda<\mu$.

Kurepa-trees are natural counterexamples to model-theoretic transfer properties. The next lemma is probably standard, but we give the proof for convenience.

28 Lemma. Let $\kappa<\lambda<\mu$ be cardinals where $\lambda$ is regular and assume that there is a $\lambda$-Kurepa-tree $T$ with at least $\mu$-many cofinal branches. Then $(\mu, \lambda) \dagger_{\rightarrow}(\lambda, \kappa)$.

Proof. Let $\mathcal{B}$ be a collection of $\mu$-many cofinal branches of $T$. The structure $(\mathcal{B}, T)$ is of type $(\mu, \lambda)$, so suppose towards a contradiction that there exists

$$
(\mathcal{A}, S) \prec(\mathcal{B}, T)
$$

where $\mathcal{A} \subseteq \mathcal{B}$ is of size $\lambda$ and $S \subseteq T$ is of size $\kappa$. Find $\delta<\lambda$ such that $S \subseteq T_{<\delta}$. Then by elementarity, every two branches in $\mathcal{A}$ split within the structure $(\mathcal{A}, S)$ which implies that $T_{\delta}$ would have size at least $|\mathcal{A}|=\lambda$. This contradicts the fact that levels of $T$ have size less than $\lambda$.

Let us point out again (cf. Section 3a) that MM is preserved by $\omega_{2}$-directed-closed forcings. This is used crucially in the proof of the next theorem.

29 Theorem. MM does not imply that $\aleph_{\omega}$ is Jonsson.

Proof. We construct a model of MM in which $\aleph_{\omega}$ is not Jonsson. First note that $\mathrm{MM}$ is consistent with $2^{\lambda}=\lambda^{+}$for all $\lambda \geq \aleph_{1}$ since this instance of the GCH can be forced with an $\omega_{2}$-directed-closed forcing. So we start with a model

$$
V \models \mathrm{MM}+2^{\lambda}=\lambda^{+} \text {for all } \lambda \geq \aleph_{1}
$$

and define a full support Easton product

$$
\mathbb{K}_{\omega}=\prod_{1<n<\omega} \mathbb{K}_{n}
$$

where $\mathbb{K}_{n}$ is the usual forcing to add an $\aleph_{n}$-Kurepa-tree with $\aleph_{\omega}$-many branches (see e.g. [11]). Note that $\mathbb{K}_{n}$ is $\aleph_{n}$-directed-closed and has the $\aleph_{n+1}$-chain condition as we assumed the arithmetic $2^{<\aleph_{n}}=\aleph_{n}$. The usual arguments for the Easton product yield that $\mathbb{K}_{\omega}$ preserves all cardinals $\aleph_{n}(n<\omega)$ and therefore preserves $\aleph_{\omega}$. It is also easy to see 
that $\mathbb{K}_{\omega}$ is an $\omega_{2}$-directed-closed forcing and hence preserves MM. By Lemma 28 we have

$$
\left(\aleph_{\omega}, \aleph_{m}\right) \not \rightarrow \quad\left(\aleph_{m}, \aleph_{n}\right) \text { for all } n<m<\omega
$$

in the generic extension $V^{\mathbb{K}_{\omega}}$. Now assume that $\aleph_{\omega}$ is Jonsson in $V^{\mathbb{K}_{\omega}}$ and use Theorem 27 . We get that $\left(\aleph_{\omega}, \aleph_{m}\right) \rightarrow\left(\aleph_{\omega}, \aleph_{n}\right)$ holds for some $n<m<\omega$. This contradicts (4.1).

The proof of Theorem 29 actually shows that $\mathrm{MM}^{++}$does not imply that $\aleph_{\omega}$ is Jonsson, where $\mathrm{MM}^{++}$means that $\omega_{1}$-many names for stationary subsets of $\omega_{1}$ can be pulled back into the ground model. We generally tried to avoid these 'plus-versions' of forcing axioms, the interested reader is referred to [2] or [19].

b. Regressive Kurepa-trees. The notion of a regressive Kurepa-tree was introduced in [9] in order to answer the question if MM is sensitive to $\omega_{2}$-closed forcings. Surprisingly, MM turned out to be sensitive to $\omega_{2}$-closed forcings but only the Namba-fragment of MM can be violated. The key notion was that of an $\omega_{1}$-regressive $\omega_{2}$-Kurepa-tree which can be added by an $\omega_{2}$-closed forcing and it was shown that MM is false once such a tree is added. We want to point out in this section that regressive Kurepa-trees have strong impact on higher versions of Chang's Conjecture even though they can be added with a very mild forcing.

30 Definition. For any tree $T$ say that the level $T_{\alpha}$ is non-stationary if there is a function $f_{\alpha}: T_{\alpha} \longrightarrow T_{<\alpha}$ which is regressive in the sense that $f_{\alpha}(x)<_{T} x$ for all $x \in T_{\alpha}$ and if $x, y \in T_{\alpha}$ are distinct then $f_{\alpha}(x)$ or $f_{\alpha}(y)$ is strictly above the meet of $x$ and $y$.

31 Definition. Let $X$ be a set of ordinals. A $\lambda$-Kurepa-tree $T$ will be called $X$-regressive if for all limit ordinals $\alpha<\lambda$ with $\operatorname{cf}(\alpha) \in X$ the level $T_{\alpha}$ is non-stationary.

The following two theorems appear in [9].

32 Theorem. For all uncountable regular $\lambda$ there is a $\lambda$-closed forcing that adds a $\lambda$-regressive $\lambda$-Kurepa-tree.

33 Theorem. Under MM, there are no $\omega_{1}$-regressive $\lambda$-Kurepa-trees for any uncountable regular $\lambda$.

A close examination of the proof of Theorem 32 actually gives:

34 Theorem. Assume $2^{<\lambda}=\lambda$ and $\kappa<\lambda<\mu$, where $\kappa$ and $\lambda$ are regular. There is a $\kappa^{+}$-directed-closed, $\lambda$-closed, $\lambda^{+}-c c$ forcing that adds a $[\kappa, \lambda)$-regressive $\lambda$-Kurepa-tree with at least $\mu$-many branches. 
Regressive Kurepa-trees are even stronger counterexamples to modeltheoretic transfer properties than the regular Kurepa-trees considered in Section 4a. This can be seen from the following Lemma.

35 Lemma. Let $\kappa<\lambda$ be regular. Assume there is a $\{\kappa\}$-regressive $\lambda$-Kurepa-tree $T$ with at least $\mu$-many branches and suppose $\kappa^{<\kappa}=\kappa$. Then $(\mu, \lambda) \nrightarrow \rightarrow\left(\kappa^{+}, \kappa\right)$.

Proof. Let $\mathcal{B}$ be the set of cofinal branches of $T$ and consider the structure $(\mathcal{B}, T)$ which is of type $(\mu, \lambda)$. Now assume towards a contradiction that $(\mu, \lambda) \rightarrow\left(\kappa^{+}, \kappa\right)$ would hold, so we find a substructure

$$
(\mathcal{A}, S) \prec(\mathcal{B}, T),
$$

where $\mathcal{A}$ has size $\kappa^{+}$and $S$ has size $\kappa$. Define $\delta=\sup ($ ht" $S)$, we have two cases:

Case 1: if $\operatorname{cf}(\delta)=\kappa$ then $T_{\delta}$ is a non-stationary level of the tree $T$. A straightforward argument using the fact that there is a regressive 1-1 function defined on $T_{\delta}$ shows that $\mathcal{A}$ has size at most $|S|=\kappa$. This is a contradiction.

Case 2: if $\operatorname{cf}(\delta)<\kappa$ then $S$ has a cofinal subtree $S_{0}$ of height $\kappa_{0}<\kappa$. Since $|S|=\kappa$, the number of branches through $S_{0}$ can not be larger than $\kappa^{\kappa_{0}} \leq \kappa^{<\kappa}=\kappa$. Again, contradiction.

36 Corollary. $M M$ is consistent with

$$
\left(\aleph_{m+1}, \aleph_{m}\right) \not \rightarrow\left(\aleph_{n+1}, \aleph_{n}\right)
$$

for all $1<n<m$ simultaneously.

Proof. Using an Easton product similar to the proof of Theorem 29: we start with a model of "MM $+2^{\lambda}=\lambda^{+}$for all $\lambda \geq \aleph_{1}$ ". Then for all $m>2$ we add an $\left[\aleph_{2}, \aleph_{m}\right)$-regressive $\aleph_{m}$-Kurepa-tree with $\aleph_{m+1}$-many branches. This product is $\omega_{2}$-directed-closed by Theorem 34 . Notice that in the extension we have $2^{<\aleph_{n}}=\aleph_{n}$ for all $1<n<\omega$. The statement of the corollary then follows from Lemma 35.

\section{REFERENCES}

[1] Joan Bagaria. Bounded forcing axioms as principles of generic absoluteness. Archive for Mathematical Logic, 39:393-401, 2000.

[2] James Baumgartner. Applications of the Proper Forcing Axiom. In K. Kunen and J.E. Vaughan, editors, Handbook of set-theoretic topology, pages 913-959. North-Holland, 1984.

[3] James Cummings, Matthew Foreman, and Menachem Magidor. Squares, scales and stationary reflection. Journal of Mathematical Logic, 1:35-98, 2001.

[4] Matthew Foreman. Games played on Boolean algebras. Journal of Symbolic Logic, 48:714-723, 1983. 
[5] Matthew Foreman, Menachem Magidor, and Saharon Shelah. Martin's Maximum, saturated ideals, and nonregular ultrafilters I. Annals of Mathematics, 127:1-47, 1988.

[6] Akihiro Kanamori. The Higher Infinite. Perspectives in Mathematical Logic. Springer-Verlag, 1997.

[7] Bernhard König. Generic compactness reformulated. Archive for Mathematical Logic, 43:311-326, 2004.

[8] Bernhard König and Yasuo Yoshinobu. Fragments of Martin's Maximum in generic extensions. Mathematical Logic Quarterly, 50:297-302, 2004.

[9] Bernhard König and Yasuo Yoshinobu. Kurepa-trees and Namba forcing. preprint, 2005.

[10] Kenneth Kunen. Saturated ideals. Journal of Symbolic Logic, 43:65-76, 1978.

[11] Kenneth Kunen. Set theory. An introduction to independence proofs. NorthHolland, 1980.

[12] Paul Larson. Separating stationary reflection principles. Journal of Symbolic Logic, 65:247-258, 2000.

[13] Justin Moore. Set mapping reflection. Journal of Mathematical Logic, 5:87-97, 2005.

[14] Saharon Shelah. On successors of singulars. In Logic Colloquium '78, volume 97 of Stud. Logic Foundations Math., pages 357-380. North-Holland, 1979.

[15] Saharon Shelah. Proper Forcing, volume 940 of Lecture notes in Mathematics. Springer-Verlag, 1982.

[16] Saharon Shelah. Proper and Improper Forcing. Perspectives in Mathematical Logic. Springer-Verlag, 1998.

[17] Stevo Todorcevic. Localized reflection and fragments of PFA. DIMACS series, 88:135-148, 2002.

[18] Boban Velickovic. Forcing axioms and stationary sets. Advances in Mathematics, 94:256-284, 1992.

[19] W. Hugh Woodin. The Axiom of Determinacy, Forcing Axioms, and the Nonstationary Ideal. Walter de Gruyter \& Co., Berlin, 1999.

[20] Yasuo Yoshinobu. Approachability and games on posets. Journal of Symbolic Logic, 68:589-606, 2003.

\author{
UNIVERSITÉ PARIS 7 \\ 2 PLACE JUSSIEU \\ 75251 PARIS CEDEX 05 \\ FRANCE
}

